\title{
Rnd3 haploinsufficient mice are predisposed to hemodynamic stress and develop apoptotic cardiomyopathy with heart failure
}

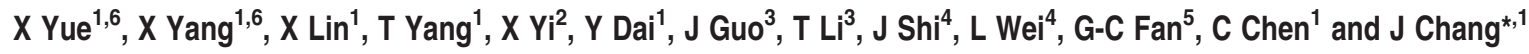

Rho family guanosine triphosphatase (GTPase) 3 (Rnd3), a member of the small Rho GTPase family, has been suggested to regulate cell actin cytoskeleton dynamics, cell migration, and apoptosis through the Rho kinase-dependent signaling pathway. The biological function of Rnd3 in the heart is unknown. The downregulation of small GTPase Rnd3 transcripts was found in patients with end-stage heart failure. The pathological significance of Rnd3 loss in the transition to heart failure remains unexplored. To investigate the functional consequence of Rnd3 downregulation and the associated molecular mechanism, we generated Rnd3 ${ }^{+I-}$ haploinsufficient mice to mimic the downregulation of Rnd3 observed in the failing human heart. Rnd3 ${ }^{+I-}$ mice were viable; however, the mice developed heart failure after pressure overload by transverse aortic constriction (TAC). Remarkable apoptosis, increased caspase-3 activity, and elevated Rho kinase activity were detected in the Rnd3 ${ }^{+I-}$ haploinsufficient animal hearts. Pharmacological inhibition of Rho kinase by fasudil treatment partially improved Rnd3 ${ }^{+I-}$ mouse cardiac functions and attenuated myocardial apoptosis. To determine if Rho-associated coiled-coil kinase 1 (ROCK1) was responsible for Rnd3 deficiency-mediated apoptotic cardiomyopathy, we established a double-knockout mouse line, the Rnd3 haploinsufficient mice with ROCK1-null background (Rnd3 $3^{+I-I R O C K 1-I-}$ ). Again, genetic deletion of ROCK1 partially but not completely rescued Rnd3 deficiency-mediated heart failure phenotype. These data suggest that downregulation of Rnd3 correlates with cardiac loss of function as in heart failure patients. Animals with Rnd3 haploinsufficiency are predisposed to hemodynamic stress. Hyperactivation of Rho kinase activity is responsible in part for the apoptotic cardiomyopathy development. Further investigation of ROCK1-independent mechanisms in Rnd3-mediated cardiac remodeling should be the focus for future study.

Cell Death and Disease (2014) 5, e1284; doi:10.1038/cddis.2014.235; published online 5 June 2014

Subject Category: Experimental Medicine

Rho family guanosine triphosphatase (GTPase) 3 (Rnd3), also called RhoE, is a member of the Rho family of GTPases. The Rho family proteins belong to the Ras superfamily of GTPases. Rnd3 was first identified as a member of the Rnd subfamily by Jeffrey Settleman's lab. ${ }^{1}$ Anne Ridley's group first suggested that Rnd3 functions as a Rho-associated coiled-coil kinase 1 (ROCK1) but not ROCK2 inhibitor. ${ }^{2}$ The current knowledge about the biological functions of Rnd3 has been mainly achieved by in vitro cell culture studies and tissue screening assays. Rnd3 binds to ROCK1 to inhibit ROCK1 downstream targets, including myosin light-chain phosphatase, myosin light chain, ${ }^{3-5}$ and LIM kinase, ${ }^{6,7}$ which are mainly involved in the regulation of cell actin cytoskeleton dynamics, cell migration, and apoptosis. ${ }^{2,6-9}$

Apoptotic cardiomyopathy is characterized by loss of cardiomyocytes, dilation of the chambers, and infiltration and maturation of fibroblasts. Apoptosis along with the activation of caspase-3 was found in the myocardium of end-stage heart failure patients. ${ }^{10-13}$ Although, levels of apoptosis detected in the failing heart are variable, ${ }^{10,14,15} \mathrm{a}$ low prevalence of apoptosis is sufficient to cause cardiac contractile depression. ${ }^{16}$ Several transgenic animal models have demonstrated that apoptotic myocyte death is a determining factor involved in the transition to failure. ${ }^{16-20}$

The knowledge of biological functions of Rnd3 in vivo is limited. Two recent mouse studies revealed an indispensable role of Rnd3 in mouse neuron development. ${ }^{21,22}$ Using a genetic deletion of Rnd3 approach, we further found that Rnd3 deficiency led to mouse hydrocephalus development. ${ }^{23}$ In this study, we extend our mouse investigation to explore the biological function of Rnd3 in the heart, which has not been studied. A significant decrease in the Rnd3 mRNA levels was

\footnotetext{
${ }^{1}$ Texas A\&M University Health Science Center, Institute of Biosciences and Technology, Houston, TX, USA; ${ }^{2}$ Department of Cardiology, Renmin Hospital of Wuhan University, Wuhan, Hubei, China; ${ }^{3}$ Cardiovascular Disease and Research Institute, Affiliated Hospital of Hainan Medical College, Haikou, Hainan, China; ${ }^{4}$ Department of Pediatrics, Indiana University School of Medicine, Indianapolis, IN, USA and ${ }^{5}$ Department of Pharmacology and Cell Biophysics, University of Cincinnati College of Medicine, Cincinnati, OH, USA

*Corresponding author: J Chang, Texas A\&M University Health Science Center, Institute of Biosciences and Technology, 2121 W. Holcombe Blvd., Houston, TX 77030, USA. Tel: + 1713677 7603; Fax: +1 713677 7410; E-mail: jchang@ibt.tamhsc.edu

${ }^{6}$ These authors contributed equally to this work.

Keywords: Rho family GTPase 3; apoptotic cardiomyopathy; heart failure; Rho kinase signaling

Abbreviations: Rnd3, Rho family GTPase 3; ROCK1, Rho-associated coiled-coil protein kinase 1; TAC, transverse aortic constriction; MYPT1, myosin phosphatase target subunit 1; MLC2 myosin light chain 2; LW, lung weight; TL, tibia length; BW, body weight

Received 08.11.13; revised 31.3.14; accepted 16.4.14; Edited by G Condorelli
} 
detected in the failing human myocardia in an early microarray screening study (Profile GDS651/212724_at/RND3 in NCBI GEO profiles). The etiologic meaning of Rnd3 downregulation in the failing human heart is unknown. The investigation of this downregulation has obvious clinical significance. To elucidate the pathological significance of Rnd3 downregulation in the heart, we established a patient-relevant mouse model, Rnd3 $^{+/-}$haploinsufficient mice. The haploinsufficient $\mathrm{Rnd}^{+/}$- mice were viable and survived to adulthood without obvious abnormalities compared to the wild-type (WT) animals under physiological conditions. However, these mice developed heart failure with apoptotic cardiomyopathy after the mice were exposed to pressure overload. Treatment by Rho kinase inhibitor or genetic deletion of ROCK1 rescued the mouse apoptotic phenotype with partial improvement of cardiac functions. We conclude that the mice with Rnd3 haploinsufficiency are predisposed to hemodynamic stress partially due to the enhanced Rho kinase activity. Our study suggests that the downregulation of Rnd3 is a risk factor in the heart, and the manipulation of Rnd3 expression could be a potential therapeutic target in human heart failure treatments.

\section{Results}

The $\mathrm{Rnd}^{+/}$- haploinsufficient mice are predisposed to pressure overload and develop severe heart failure after the transverse aortic constriction challenge. The $\mathrm{Rnd} 3^{+/-}$ mice were viable without detectable abnormalities in heart morphology compared to WT mice (Figure 1a, sham and Supplementary Table 1). The cardiac functions evaluated by echocardiography showed no significant differences between the two groups of mice (Figure 1b, sham and Supplementary Table 1). However, obvious heart enlargement and dilation of ventricular chambers were observed in the Rnd3 haploinsufficient heart after hemodynamic stress by transverse aortic constriction (TAC; Figure 1a, TAC). The ejection fraction (EF) and fractional shortening (FS) in the haploinsufficient hearts were dramatically decreased from 66.7 and $36.8 \%$ to 15.1 and $6.7 \%$, respectively (Figure $1 \mathrm{~b}$ ). Consistent with the echo analysis in the $\mathrm{Rnd}^{+/-}$mice, the animal lung weight over body weight (LW/BW) and the LW over tibia length (LW/TL) were significantly increased (Figure 1c), indicating heart failure development.

Significant increases in apoptosis and Rho kinase activity in the $\mathrm{Rnd}^{+/-}$haploinsufficient hearts after TAC. To assess the Rnd $3^{+/-}$failing animal hearts induced by TAC, we conducted terminal deoxynucleotidyl transferase dUTP nick end labeling (TUNEL) staining and immunoblot analysis for caspase-3. Significant amounts of TUNEL-positive cells were observed in the Rnd3 haploinsufficient heart compared to the WT control heart (Figures 2a and $b$ ). The higher caspase-3 activity was also detected in the same cohort of the Rnd $3^{+/}$hearts when compared to the WT controls (Figures $2 \mathrm{c}$ and d). Meanwhile, we evaluated the Rho kinase activity in the mutant hearts by immunoblotting for the phosphorylation of myosin phosphatase target subunit 1 (MYPT1). Hyperphosphorylation of MYPT1 was observed, indicating an elevated Rho kinase activity in the Rnd3 $^{+/}$- hearts (Figures $2 e$ and f). a
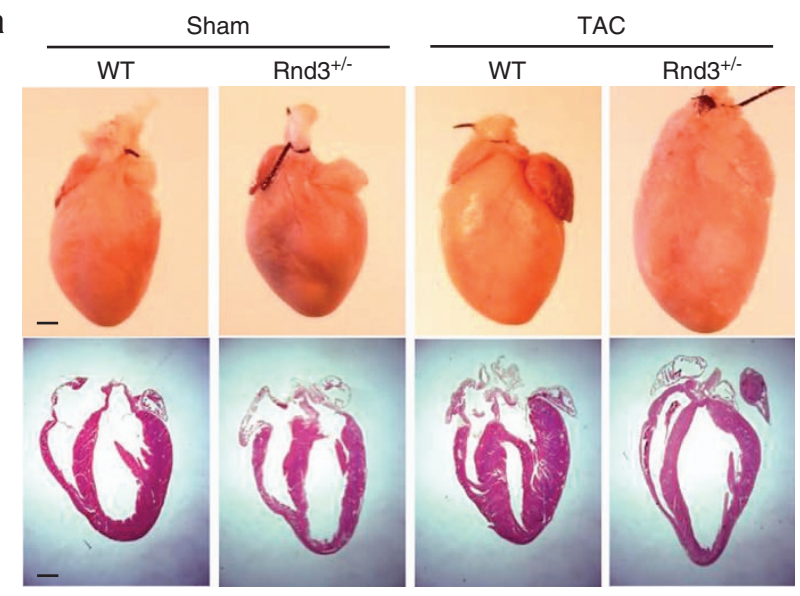

b
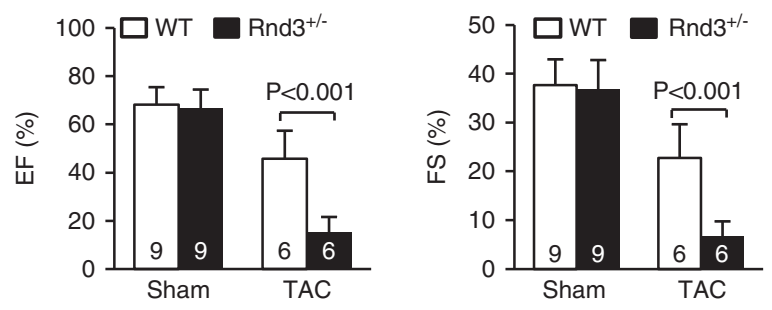

C
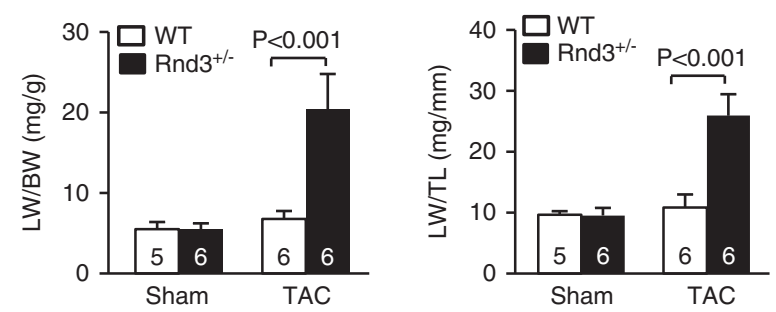

Figure $1 \mathrm{Rnd}^{+/}$- haploinsufficient mice developed heart failure after 3-week TAC. (a) Representative pictures of whole animal hearts and their hematoxylin and eosin staining sections. Enlarged ventricles were displayed in the Rnd $3^{+1-}$ mutant hearts after TAC. (b) Significant decreases in the cardiac EF and FS were detected in Rnd3 ${ }^{+1}$ mice compared to the WT mice after TAC. (c) Significant increases in LW over BW and LW over TL indicated heart failure development in $\mathrm{Rnd}^{+l}$ hearts by pressure overload. The numbers in the columns represent the number of mice in each group. Statistical significance was determined by one-way ANOVA followed by Student-Newman-Keuls method. Data are means \pm S.D. Scale bar represents $1 \mathrm{~mm}$. BW, body weight; EF, ejection fraction; FS, fractional shortening; LW, lung weight; TAC, transverse aortic constriction; TL, tibia length; WT, wild-type

Rnd3 deficiency is sufficient to induce apoptotic cardiomyopathy along with activation of Rho kinase signaling. We recently demonstrated that the $\mathrm{Rnd} 3^{-1-}$ homozygous mice were embryonically lethal around E11.0. The embryonic mice with Rnd3 deficiency developed heart failure. ${ }^{24}$ To investigate if the Rnd3 deficiency directly leads to apoptotic cardiomyopathy under physiological conditions, we analyzed E10.5 Rnd3 ${ }^{-1-}$ homozygous mouse hearts. Significant amounts of TUNEL-positive cells and elevated caspase-3 activity were observed in the Rnd3-null hearts compared to the WT control hearts (Figures 3a-c). Hyperphosphorylation was detected in the two targets of ROCK1, MYPT1 and myosin light chain 2 (MLC2), indicating an increase in Rho kinase activity in the Rnd3-null hearts (Figure 3d). 
a

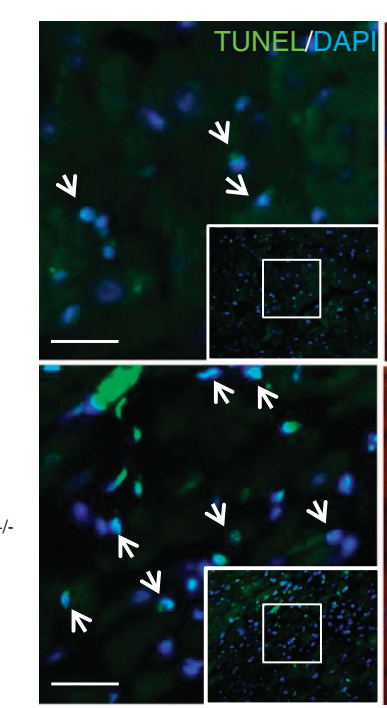

c

c

e

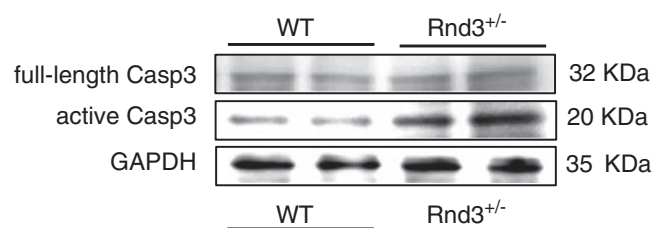

p-T853 MYPT1

MYPT1

ROCK1

GAPDH

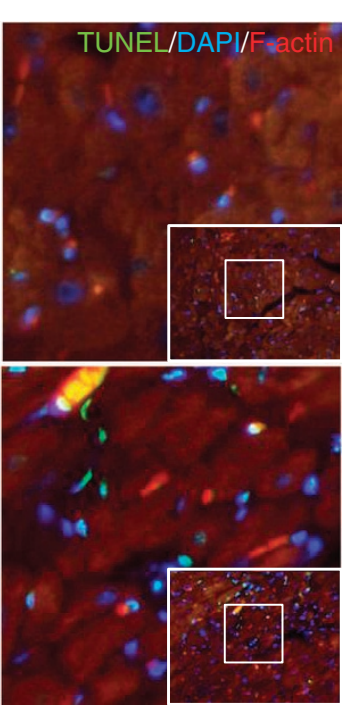

Rnd3+- b

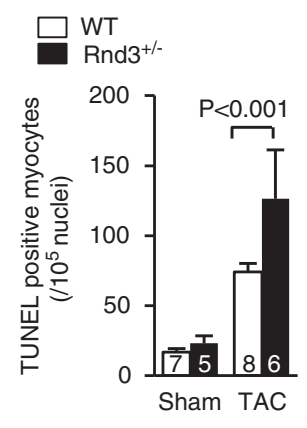

d

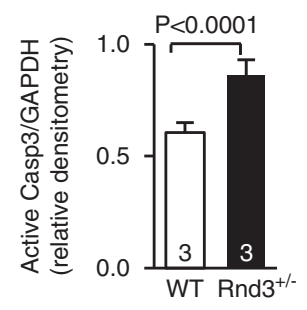

f

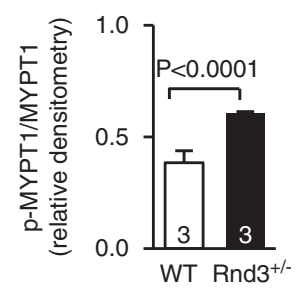

Figure $2 \mathrm{Rnd}^{+1}$ - haploinsufficient mice were predisposed to hemodynamic stress. Severe cardiac apoptosis and Rho kinase activation were detected after TAC. (a) Significant increases in TUNEL-positive cells were observed in Rnd3 ${ }^{+l}$ - hearts compared to WT control hearts after TAC. The arrows indicate TUNEL-positive cells (green) overlapped with nucleus counter-staining (blue). Cardiomyocytes were visualized by red fluorescent staining for F-actin. Scale bar represents $10 \mu \mathrm{m}$. (b) Quantification of the TUNEL-positive cells in mouse hearts. Statistical significance was determined by one-way ANOVA followed by Student-Newman-Keuls method. Data are means \pm S.D. (c) Higher caspase-3 activities were detected in Rnd $3^{+1-}$ hearts compared to WT animal hearts after TAC. (d) Densitometry analysis of active caspase-3 normalized by GAPDH. (e) Hyperphosphorylation of Rho kinase substrate MYPT1 exhibited in Rnd $3^{+1}$ - hearts indicates elevated Rho kinase activity compared to WT hearts after TAC. (f) Densitometry analysis of MYPT1 phosphorylation. Statistical significance was determined by unpaired, two-tailed Student's $t$-test. Data are means \pm S.D. Casp3, caspase-3; GAPDH, glyceraldehyde 3-phosphate dehydrogenase; MYPT1, myosin phosphatase target subunit; ROCK1, Rho-associated coiled-coil kinase 1; TUNEL, terminal deoxynucleotidyl transferase dUTP nick end labeling. The numbers in the columns represent the number of mice in each group

Heart failure and apoptotic cardiomyopathy are partially rescued by treatment with Rho kinase inhibitor fasudil in Rnd3 $^{+} I_{-}$haploinsufficient mice. Rnd3 has been suggested to function as a Rho kinase ROCK1 inhibitor. Rnd3 haploinsufficient mice develop heart failure along with elevated Rho kinase activity after pressure overload. To explore a possible mechanism that Rnd3 downregulationmediated cardiac dysfunction is associated with increased Rho kinase activity, we treated the $\mathrm{Rnd} 3^{+/}$mice with fasudil, a clinically used Rho kinase inhibitor, immediately after TAC. Three weeks later, significant improvements in cardiac functions (Figure 4a) and less cardiac apoptosis (Figures $4 \mathrm{~b}$ and $\mathrm{c}$ ) were observed in fasudil-treated mice compared to non-treated animals. Cardiac EF and FS were improved from 19.1 and $8.6 \%$ to 40.3 and $19.5 \%$, respectively (Figure 4a). The increases in caspase-3 activity (Figures 4d and e) and Rho kinase activity (Figures $4 \mathrm{f}$ and $\mathrm{g}$ ) were also significantly eased. To rule out if the improvement of cardiac phenotype was due to fasudil-mediated vasodilation, mouse blood pressures were compared among sham, TAC, and TAC plus fasudil groups in WT and
$\mathrm{Rnd}^{+/}$- haploinsufficient mice. We found no significant differences in blood pressures among the groups or between WT and $\mathrm{Rnd}^{+/-}$haploinsufficient mice, suggesting a minimal effect of vasodilation on the cardiac improvement (Supplementary Figure 1).

Although, the cardiac apoptosis and Rho kinase activity returned to the control levels, levels of the improved cardiac EF and FS were still significantly below the levels in sham control mice (Figure 4a). The cardiac functional recoveries by Rho kinase inhibitor treatment were partial, suggesting that other factors besides Rho kinase activation contribute to Rnd3 deficiency-mediated cardiac dysfunction as well.

Genetic deletion of ROCK1 attenuates Rnd3 deficiencymediated apoptotic cardiomyopathy and improves cardiac functions. To further investigate the role of ROCK1 activation in Rnd3 deficiency-mediated cardiomyopathy, we created the Rnd3 ${ }^{+/-}$with ROCK1-null background mouse line, Rnd3 ${ }^{+/-/ R O C K 1-/-}$ (Figure 5a). The double-knockout mice showed normal cardiac EF and FS (Figure 5b, sham). The Rnd $3^{+/-}$mice and Rnd3 $3^{+/-/ R O C K 1-/-}$ mice were then 
Red: F-actin staining

Blue: DAPI staining

Green: TUNEL staining
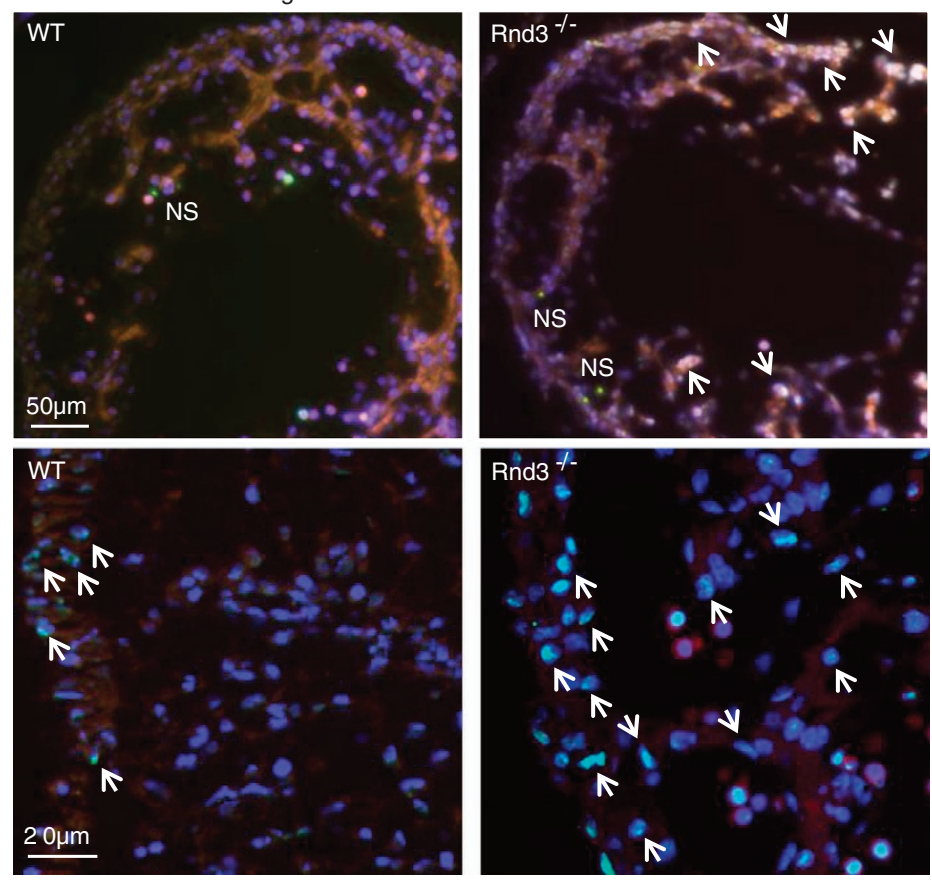

b

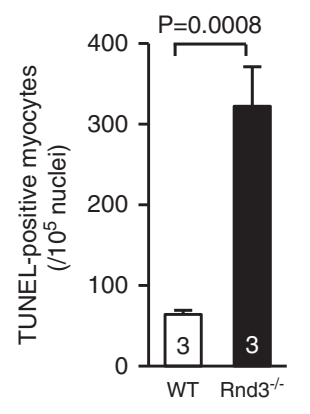

C
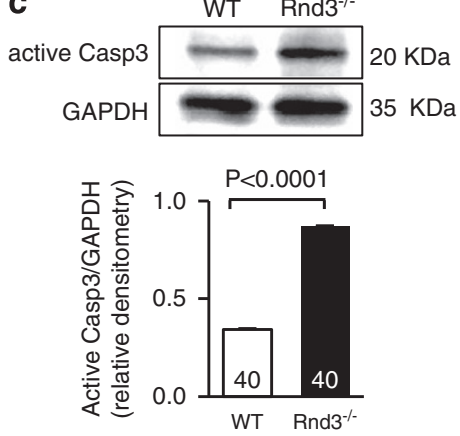

d
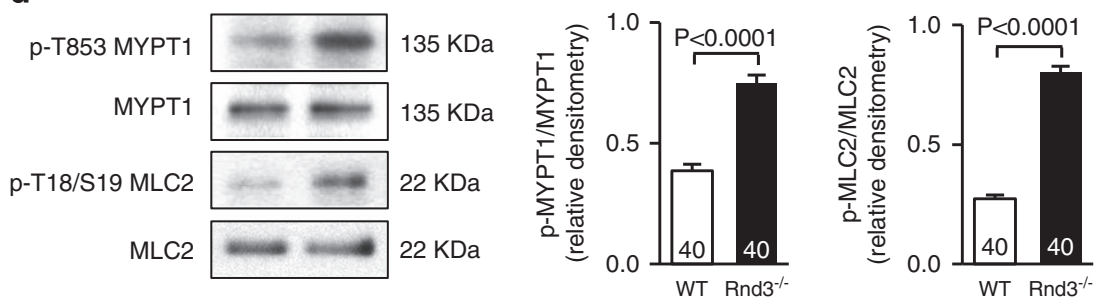

Figure 3 Complete deletion of Rnd3 gene resulted in severe apoptotic cardiomyopathy along with the elevation of Rho kinase activity in the mouse hearts. (a) A significant amount of TUNEL-positive cells were displayed in E10.5 Rnd3-null hearts. The arrows indicate TUNEL-positive cells (green) overlapped with nucleus counter-staining. Cardiomyocytes were visualized by red fluorescent staining for F-actin. NS indicates nonspecific staining. (b) Quantification of the TUNEL-positive cells in hearts. (c) Elevated caspase-3 activity was detected in Rnd3-null hearts by immunoblot analyses and quantified by immunoblot densitometry analyses. (d) Elevated Rho kinase activity was detected in Rnd3-null hearts by immunoblotting for two Rho kinase substrates, MYPT1 and MLC2. The immunoblot densitometry analyses were quantified. Statistical significance was determined by unpaired, two-tailed Student's $t$-test. Data are means \pm S.D. TUNEL, terminal deoxynucleotidyl transferase dUTP nick end labeling; MLC2, myosin light chain 2. The numbers in the columns represent the number of mice in each group

exposed to TAC stress for 3 weeks followed by cardiac function analysis and cardiac apoptosis assessment. Again, we observed a deteriorated cardiac function with EF $19.9 \%$ and FS $8.9 \%$ in Rnd3 ${ }^{+/-}$haploinsufficient mice after TAC. However, double-knockout mice showed better cardiac functions with EF $52.9 \%$ and FS $26.5 \%$ compared to $\mathrm{Rnd}^{+1-}$ mice (Figure 5b, TAC). Less apoptotic cells and attenuated caspase-3 activity were detected in double-knockout mice compared to Rnd $3^{+/}$mice (Figures $5 c-f$ ), suggesting that ROCK1 activation contributes to Rnd3 deficiency-mediated 
a
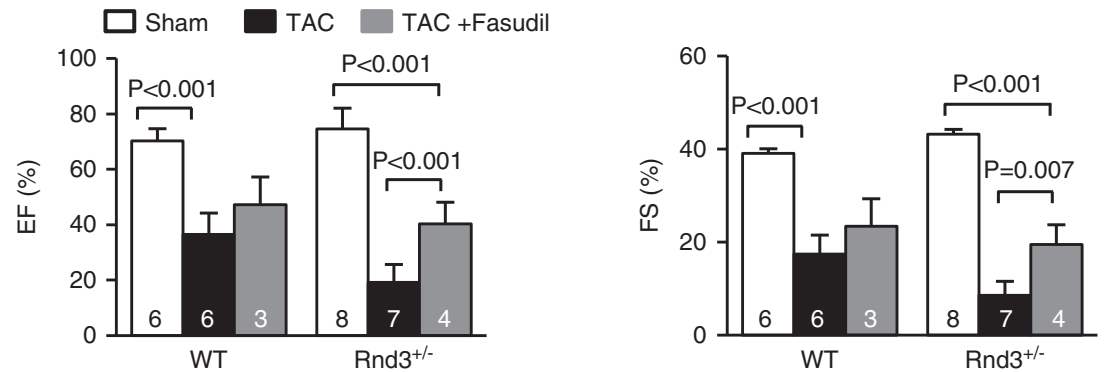

b
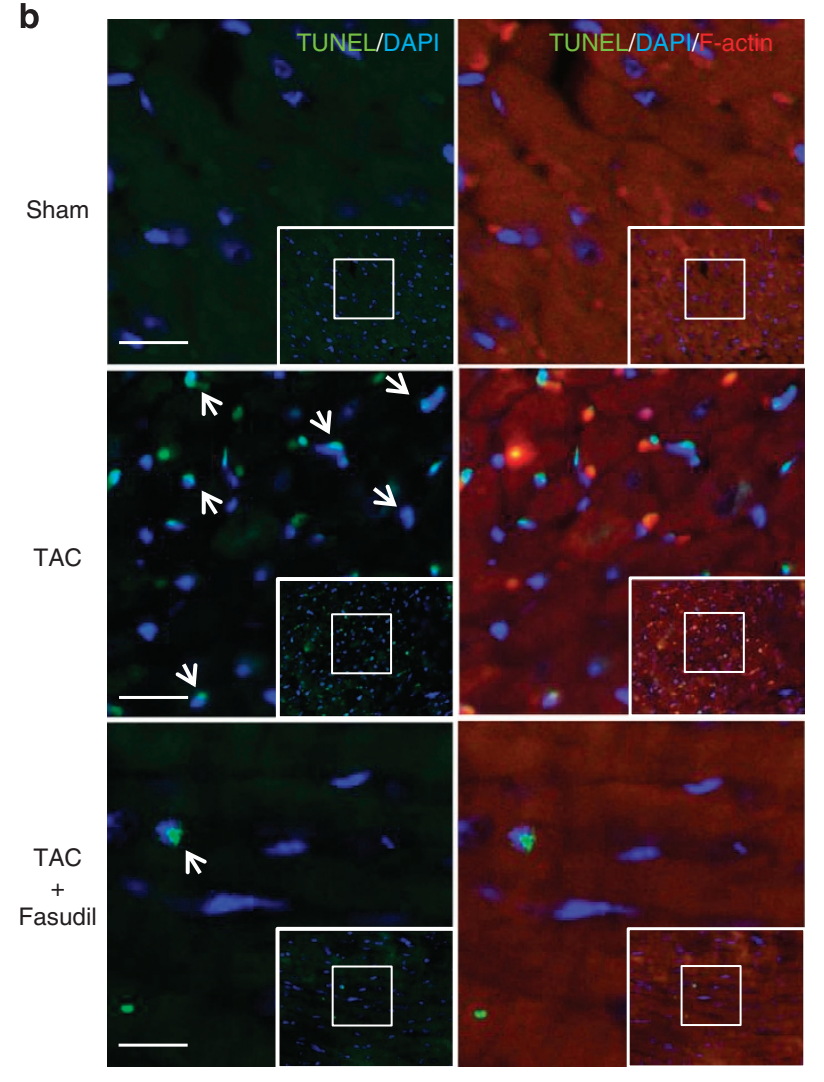

L/DAPI/E
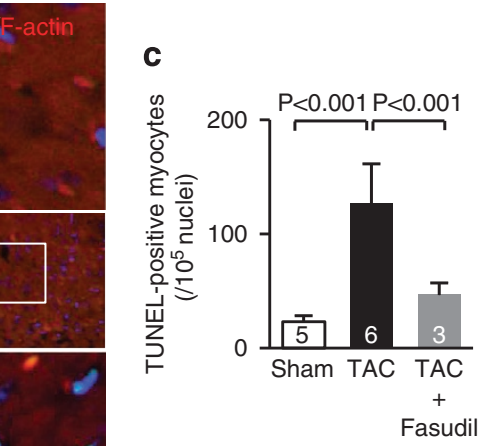

d
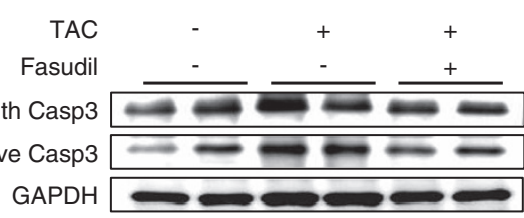

$32 \mathrm{KDa}$

full-length Casp3

active Casp3

GAPDH

$20 \mathrm{KDa}$ $35 \mathrm{KDa}$

f

TAC

Fasudil

p-T853 MYPT1

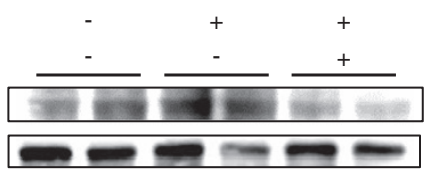

$135 \mathrm{KDa}$

MYPT1

$135 \mathrm{KDa}$

g

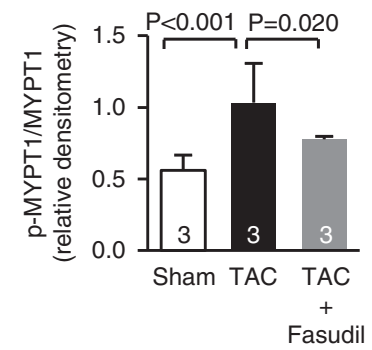

Figure 4 Rho kinase inhibitor fasudil treatment partially improved Rnd3 ${ }^{+/}$mouse cardiac functions and attenuated apoptotic cardiomyopathy induced by 3-week TAC. (a) Echocardiographic assessment of cardiac functions. (b) A comparison of TUNEL staining in Rnd3 haploinsufficient heart sections among three groups: Sham, TAC only, and TAC plus fasudil treatment. The arrows indicate TUNEL-positive cells (green) overlapped with nucleus counter-staining. Cardiomyocytes were visualized by red fluorescent staining for F-actin. Scale bar represents $10 \mu \mathrm{m}$. (c) Quantification of TUNEL staining among the hearts from the three groups. (d) Active and full-length of caspase-3 expression levels among the three groups assessed by immunoblot. (e) Densitometry analysis of active caspase-3 normalized by GAPDH. (f) Phosphorylation levels of Rho kinase substrate MYPT1 among the three groups assessed by immunoblot. (g) Densitometry analysis of MYPT1 phosphorylation. Statistical significance was determined by one-way ANOVA followed by Student-Newman-Keuls method. Data are means \pm S.D. The number in each column represents the number of mice in the group 
a

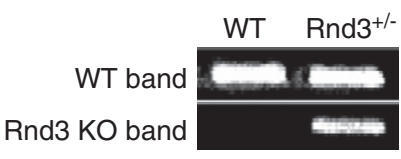

b

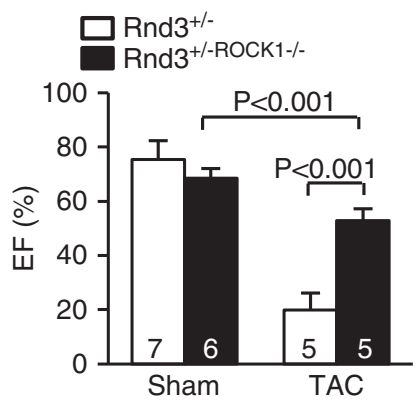

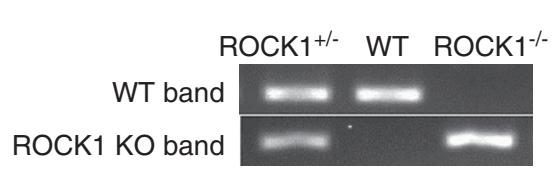

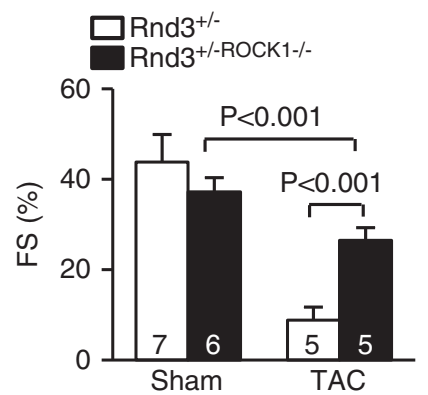

c
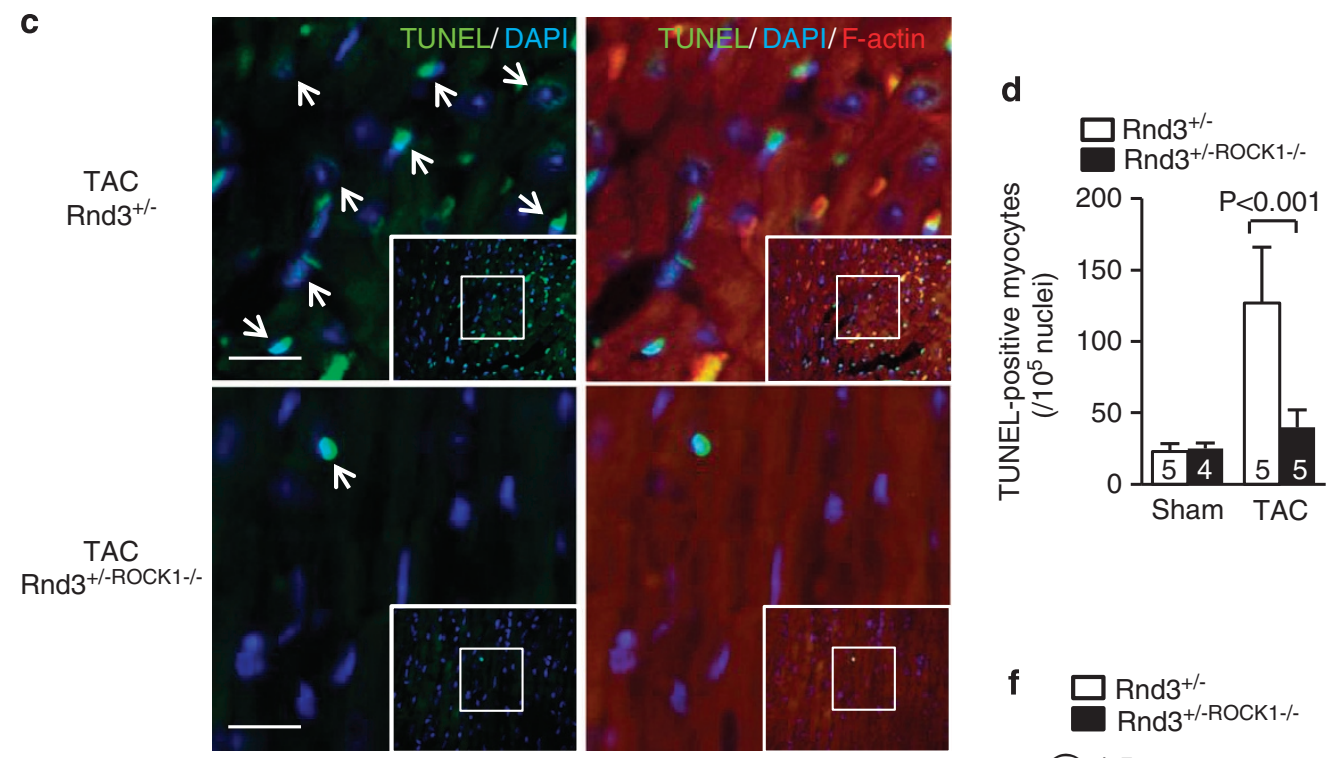

e

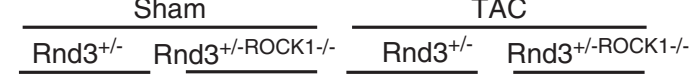
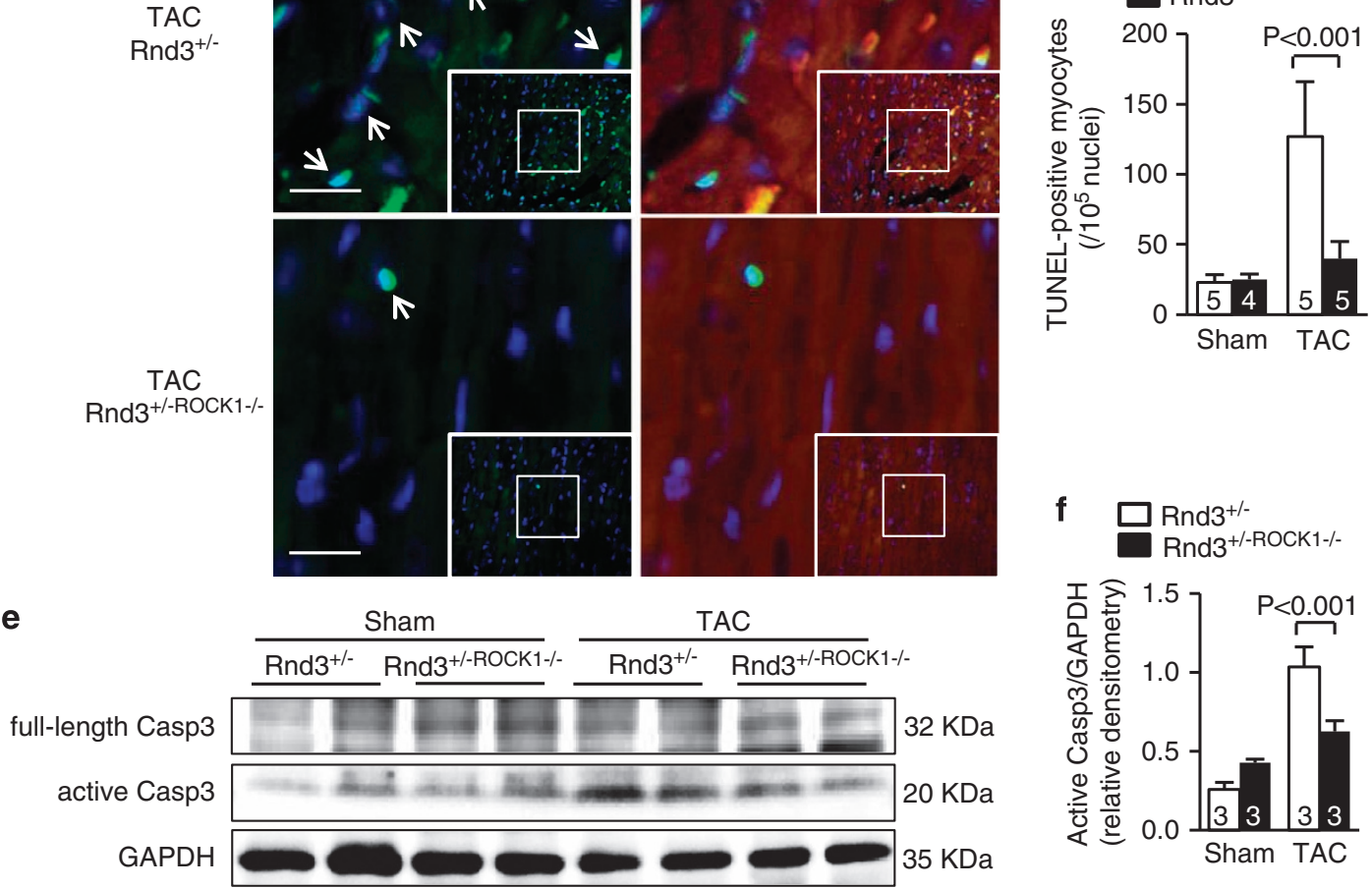

Figure 5 Genetic deletion of Rho kinase ROCK1 partially improved Rnd3 ${ }^{+1-}$ mouse cardiac functions and attenuated apoptotic cardiomyopathy induced by TAC. (a) The genotyping profile showed the establishment of the double-knockout (DK) mouse line, Rnd3 ${ }^{+I-R O C K 1-I-}$. (b) Echocardiographic assessment of cardiac functions. (c) A comparison of TUNEL staining between the Rnd3 ${ }^{+}-$hearts and DK mouse hearts. The arrows indicate TUNEL-positive cells (green) overlapped with nucleus counterstaining. Cardiomyocytes were visualized by red fluorescent staining for F-actin. Scale bar represents $10 \mu \mathrm{m}$. (d) The quantification of the TUNEL results between the two groups of mouse hearts. (e) Active and full-length of caspase-3 expression levels between the two groups of mouse hearts assessed by immunoblot. (f) Densitometry analysis of active caspase-3 normalized by GAPDH. Statistical significance was determined by one-way ANOVA followed by Student-Newman-Keuls method. Data are means \pm S.D. The number in each column represents the number of mice in the group. KO, knockout

apoptotic cardiomyopathy. However, consistent with Rho kinase inhibitor fasudil treatment, genetic deletion of ROCK1 was not able to completely rescue Rnd3 deficiency-mediated cardiac dysfunction (Figure 5b).

\section{Discussion}

The direct role of Rnd3 in the heart remains unexplored. The current paradigm, derived from the majority of the published studies on Rnd3, is that Rnd3 functions as an inhibitor of Rho signaling by either directly binding to ROCK1 or indirectly targeting RhoA through p190-B RhoGAP. 2,8,25,26 Overexpression of Rnd3 inhibited ROCK1-mediated biological effects, and the reduced expression of Rnd3 potentiated ROCK1 activity. ${ }^{2,8,27}$ Therefore, the functions of Rnd3 have been almost entirely linked to cell actin cytoskeleton dynamics, cell migration, and apoptosis demonstrated by in vitro cell culture. ${ }^{2,6-9}$ Recently, emerging evidence starts to 
show that Rnd3 may be involved in the regulation of ERK and p38 pro-survival pathway in U87 cells and keratinocytes; ${ }^{28,29}$ while in prostate cancer cell lines, the effect of Rnd3 on cell apoptosis was associated with cell cycle regulation. ${ }^{30}$ Our recent Rnd3 genetic mouse study demonstrated that Rnd3 is a novel mediator that inhibits Notch signaling by facilitating the ubiquitination of the Notch active isoform, NICD, in the mouse brain. Genetic deletion of $R$ nd3 results in hyperactivation of Notch signaling. ${ }^{23}$ The new biological function of Rnd3 was supported by two recent studies showing an indispensable role of Rnd3 in animal brain development. ${ }^{21,22}$ These emerging cell and animal studies strongly suggest that Rnd3 has much broader biological functions than previously thought, which are beyond the Rho signaling pathway.

In this study, we provide evidence to support this new concept. We demonstrate that the integrity of Rnd3 gene expression is critical in the heart in response to hemodynamic stress. The downregulation of Rnd3 predisposes the animal heart to pressure overload. We reveal that hyperactivation of Rho kinase is part of the mechanisms responsible for Rnd3 deficiency-induced apoptotic cardiomyopathy. Pharmacological inhibition of Rho kinase or genetic silence of ROCK1 partially improves $\mathrm{Rnd}^{+/}$mouse cardiac function and attenuates cardiac apoptosis. Further investigation of Rho kinase-independent regulatory mechanism of Rnd3 in the heart will provide a new strategy for heart failure treatment.

The pro-apoptotic effect of ROCK1 has been characterized in our previous studies in mice as well as others in cell lines. ${ }^{31-34}$ By loss-of-function approaches, we and other groups found that genetic deletion of the ROCK1 gene enhanced mouse heart resistance against stress-induced cardiac apoptosis and fibrosis, and preserved cardiac functions. ${ }^{35-38}$ In human patients with end-stage heart failure, we found a significant increase in Rho kinase activity in the failing myocardium. This increase was directly associated with caspase-3-dependent cleavage of ROCK1, which generated a constitutively active Rho kinase subspecies. The truncated ROCK1 facilitated caspase-3 activation and fibrotic gene upregulation, which led to a feed-forward loop promoting myocyte apoptosis and heart failure. $^{31,35}$

Rho kinase is a downstream mediator of RhoA, and is believed to play a critical role in mediating the effects of RhoA on stress fiber formation, smooth muscle contraction, cell adhesion, membrane ruffling, and cell motility. ${ }^{27,39}$ Studies of regulatory activation of ROCK1 discovered that Rnd3 inhibits RhoA-mediated ROCK1 signaling by specifically binding to ROCK1 but not ROCK2. ${ }^{2,8}$ Rnd3 is one of the Rnd subsets of the Rho family proteins, and is the only endogenous ROCK1 antagonist found so arr. $^{26}$ In contrast to the canonical mode of Rho family proteins, the Rnd subfamily members, including Rnd1, Rnd2, and Rnd3, bind but do not hydrolyze GTP. They are defective in GTPase activity even in the presence of RhoGAPs. ${ }^{1,40-42}$ Limited data on the biological effect of Rnd3 on ROCK1 have been obtained via the gain- and loss-offunction approaches in vitro. Overexpression of Rnd3 inhibited ROCK1-mediated biological effects, including stress fiber formation, phosphorylation of myosin light-chain phosphatase, and apoptosis. Reduced expression of Rnd3 promoted cell apoptosis by potentiating ROCK1 activity. ${ }^{2,8,27}$ The antiapoptotic effect of Rnd3 was observed in response to genotoxic stress; ${ }^{8}$ chemotherapeutic agents or ultraviolet B radiation-induced DNA damage in different cell lines. ${ }^{28,43}$ Reduced expression levels of Rnd3 in different cancers were observed, and were proposed to associate with tumor invasion, apoptosis, and metastasis. ${ }^{30,44-46}$

Fasudil is the only clinically available Rho kinase inhibitor, and it has been in clinical use as an antivasospasm agent in Japan since 1995. Several clinical trials have demonstrated that fasudil increased forearm blood flow in hypertensive patients, ${ }^{47,48}$ attenuated patients' coronary artery spasms, ${ }^{49}$ improved coronary microcirculation, ${ }^{50}$ and increased the ischemic threshold of angina patients during exercise with a trend toward increased exercise duration. ${ }^{51}$ Like any other pharmacological inhibitors, the inhibitory effect of fasudil on other protein kinases, such as PKA, has also been reported at high dosage applications. To eliminate any potential side effects of fasudil and to validate a direct involvement of ROCK1 in Rnd3-mediated cardiomyopathy, ROCK-null background mice with Rnd3 haploinsufficiency were introduced. Consistent with the Rho kinase treatment result, we found that genetic loss of ROCK1 gene also partially attenuated hemodynamic stress-induced cardiomyopathy in Rnd3 haploinsufficient mice. The data provide definitive evidence that Rho kinase activation is responsible in part for Rnd3 deficiency-induced cardiomyopathy.

The relationship between Rnd3 protein expression levels and its significance in human heart failure remains unclear. $A$ downregulation of $\mathrm{Rnd}$ 3 transcripts was reported in one failing human myocardium microarray database. The pathological consequence of the reduced Rnd3 mRNA in heart failure patients is also unknown. We took advantage of the haploinsufficient mice to investigate the pathological role of Rnd3 downregulation in the animal. From this study, we conclude that a lower expression level of Rnd3 is a deleterious factor in the heart.

In summary, our study demonstrates an essential role of Rnd3 for normal cardiac functions in mice. Genetic loss of Rnd3 predisposes the animal heart to transition to failure. Activation of Rho kinase signaling is partially responsible for this maladaptive transition. Our findings provide a potential alternative target for pharmacological manipulations in heart failure treatment.

\section{Materials and Methods \\ Generation and verification of Rnd3 knockout (KO) mice and Rnd3 haploinsufficient with ROCK1-null double-knockout Rnd3 $^{+l-/ \text { ROCK1 } 1-l-}$ mice (DK). The establishment of individual Rnd3 $\mathrm{KO}$ and ROCK1 KO mouse lines were described previously. ${ }^{23,36}$ We bred Rnd3 KO mice with ROCK1 KO mice to achieve Rnd $3^{+/-/ R O C K 1-1-}$ DK mice. All animal experiments were approved by the Institutional Animal Care and Use Committee of the Texas A\&M University Health Science Center-Houston.}

TAC and fasudil treatment. TAC was conducted in 10-15-week-old adult male mice. The thymus was retracted to expose the transverse aorta. Between the right innominate and left carotid artery, an aortic constriction was achieved by tying a $6-0$ suture against a $3 \mathrm{~mm}$ length of 27 gauge needle. After two knots, the 27 gauge needle was promptly removed, which yielded a constriction of $\sim 0.3 \mathrm{~mm}$ as the outer diameter of the 27 gauge needle. The procedure imposed a $60-80 \%$ aortic constriction on the animal. To minimize the variation of the constriction among the animals, only the mice with a ratio of right-to-left carotid artery flow velocity from $5: 1$ to $10: 1$ were used. The artery flow velocity was measured by a Pulsed Doppler (Indus Instruments, Houston, TX, USA). As a control, a sham 
operation without occlusion was performed on age-matched littermate mice. For the rescue experiment, mice were allowed access to drinking water containing fasudil ( $\sim 100 \mathrm{mg} / \mathrm{kg} /$ day; LC Laboratories, Woburn, MA, USA) for 3 weeks starting immediately after TAC surgery. All of the TAC-induced pressure overload stress lasted for 3 weeks.

Cardiac function assessment by echocardiography and blood pressure measurement. For cardiovascular measurements, a Vevo770 High-Resolution Micro-Imaging System (VisualSonics, Toronto, ON, Canada) with $30-\mathrm{MHz}$ probe (RMV-707B) was used as previously described. ${ }^{35}$ Male mice were anesthetized with 3-4\% isoflurane, and then switched to $1-1.5 \%$ isoflurane mixed with $100 \%$ oxygen. At the level of the papillary muscles, two-dimensional-guided M-mode echocardiography was obtained from anterior (+ septum) and posterior walls. Mouse systolic blood pressure measurement was recorded by Mouse Blood Pressure System (MRBP-MMC, IITC Life Science Inc, CA, USA) through tail-cuff method after the mouse was trained for 2-3 days until it was comfortable and quiet in the nose cone animal holder. Mouse diastolic blood pressure was calculated by the software MRBP Monitor Version 1.59 (MRBP-MMC, IITC Life Science Inc, CA, USA).

Immunoblotting, hematoxylin and eosin staining, and fluorescence staining. Protein samples for western blot analysis were extracted and separated as described previously. ${ }^{52}$ Commercially available antibodies were from the following sources: MYPT1 (2634S), Phospho-T853 MYPT1 (4563S) from Cell Signaling Technology (MA, USA); MLC2 (PA5-17624), Phospho-Thr18/Ser19 MLC (PA5-17727) from Thermo Scientific Pierce (IL, USA); caspase-3 (sc-7148) from Santa Cruz Biotechnology (CA, USA). Equivalent protein loading was verified by the intensity of the GAPDH blot.

Hearts were fixed using the Langendorff perfusion system (Radnoti, 120108). Paraffin sections of the whole heart were used for hematoxylin and eosin staining and TUNEL staining (In Situ Cell Death Detection Kit, 11684795910, Roche, USA). Pictures were taken under the $\times 40$ microscope objective. A total of 20 staining pictures from each group (3-8 mice) were quantified for TUNEL analysis by Leica Application Suite Imaging Software (Version 4.0, Germany). F-actin and nuclei were visualized by phalloidin conjugated to Alexa Fluor 594 (A12381, Life Technologies, Grand Island, NY, USA) and DAPI staining, respectively. The images were acquired by fluorescence microscopy.

Statistical analysis. Data are expressed as means \pm S.D. In multiple group comparisons, one-way ANOVA followed by Student-Newman-Keuls method was used. In two group comparisons, unpaired, two-tailed Student's $t$-test was used. All of the analyses were conducted by SigmaPlot 11.0 (Systat, San Jose, CA, USA). A value of $P<0.05$ was considered statistically significant.

\section{Conflict of Interest}

The authors declare no conflict of interest.

Acknowledgements. We thank Ms. Kelsey Andrade for editorial assistance to the manuscript. This work was supported by the China Scholarship Council (X Yue); the AHA Postdoctoral Fellowship 13POST17260043 (X Yang); National Natural Science Foundation of China 81000073, 81160020, Key Project of Chinese Ministry of Education 212137, and HJHZ2013-06 (JG); Key Program of Science and Technology of Hainan Province ZDXM20100045 (TL); NIH-NHLBI R01 HL-087861 (G-CF); and the NIH-NHLBI R01HL102314, R21HL094844 and K02HL098956 (JC).

1. Foster R, Hu KQ, Lu Y, Nolan KM, Thissen J, Settleman J. Identification of a novel human Rho protein with unusual properties: GTPase deficiency and in vivo farnesylation. Mol Cell Biol 1996; 16: 2689-2699.

2. Riento K, Guasch RM, Garg R, Jin B, Ridley AJ. RhoE binds to ROCK I and inhibits downstream signaling. Mol Cell Biol 2003; 23: 4219-4229.

3. Amano M, Chihara K, Kimura K, Fukata Y, Nakamura N, Matsuura $Y$ et al. Formation of actin stress fibers and focal adhesions enhanced by Rho-kinase. Science 1997; 275: 1308-1311.

4. Kimura K, Ito M, Amano M, Chihara K, Fukata Y, Nakafuku M et al. Regulation of myosin phosphatase by Rho and Rho-associated kinase (Rho-kinase). Science 1996; 273 : 245-248.

5. Leung T, Chen XQ, Manser E, Lim L. The p160 RhoA-binding kinase ROK alpha is a member of a kinase family and is involved in the reorganization of the cytoskeleton. $\mathrm{Mol}$ Cell Biol 1996; 16: 5313-5327.
6. Maekawa M, Ishizaki T, Boku S, Watanabe N, Fujita A, Iwamatsu A et al. Signaling from Rho to the actin cytoskeleton through protein kinases ROCK and LIM-kinase. Science 1999; 285: 895-898

7. Arber S, Barbayannis FA, Hanser H, Schneider C, Stanyon CA, Bernard $\mathrm{O}$ et al. Regulation of actin dynamics through phosphorylation of cofilin by LIM-kinase. Nature 1998; 393: 805-809.

8. Ongusaha PP, Kim HG, Boswell SA, Ridley AJ, Der CJ, Dotto GP et al. RhoE is a pro-survival p53 target gene that inhibits ROCK I-mediated apoptosis in response to genotoxic stress. Curr Biol 2006; 16: 2466-2472.

9. Villalonga P, Guasch RM, Riento K, Ridley AJ. RhoE inhibits cell cycle progression and Ras-induced transformation. Mol Cell Biol 2004; 24: 7829-7840.

10. Narula J, Haider N, Virmani R, DiSalvo TG, Kolodgie FD, Hajjar RJ et al. Apoptosis in myocytes in end-stage heart failure. N Engl J Med 1996; 335: 1182-1189.

11. Narula J, Pandey P, Arbustini E, Haider N, Narula N, Kolodgie FD et al. Apoptosis in heart failure: release of cytochrome $c$ from mitochondria and activation of caspase-3 in human cardiomyopathy. Proc Natl Acad Sci USA 1999; 96: 8144-8149.

12. Blankenberg F, Narula J, Strauss HW. In vivo detection of apoptotic cell death: a necessary measurement for evaluating therapy for myocarditis, ischemia, and heart failure. $J \mathrm{NuCl}$ Cardiol 1999; 6: 531-539.

13. Elsasser A, Suzuki K, Schaper J. Unresolved issues regarding the role of apoptosis in the pathogenesis of ischemic injury and heart failure. J Mol Cell Cardiol 2000; 32: 711-724.

14. Olivetti G, Abbi R, Quaini F, Kajstura J, Cheng W, Nitahara JA et al. Apoptosis in the failing human heart. N Engl J Med 1997; 336: 1131-1141.

15. Reed JC, Paternostro G. Postmitochondrial regulation of apoptosis during heart failure. Proc Natl Acad Sci USA 1999; 96: 7614-7616.

16. Wencker D, Chandra M, Nguyen K, Miao W, Garantziotis S, Factor SM et al. A mechanistic role for cardiac myocyte apoptosis in heart failure. J Clin Invest 2003; 111: 1497-1504.

17. Nadal-Ginard B, Kajstura J, Anversa P, Leri A. A matter of life and death: cardiac myocyte apoptosis and regeneration. J Clin Invest 2003; 111: 1457-1459.

18. Ueno S, Ohki R, Hashimoto T, Takizawa T, Takeuchi K, Yamashita Y et al. DNA microarray analysis of in vivo progression mechanism of heart failure. Biochem Biophys Res Commun 2003; 307: 771-777.

19. Narula J, Arbustini E, Chandrashekhar Y, Schwaiger M. Apoptosis and the systolic dysfunction in congestive heart failure. Story of apoptosis interruptus and zombie myocytes. Cardiol Clin 2001; 19: 113-126.

20. Kitsis RN, Mann DL. Apoptosis and the heart: a decade of progress. J Mol Cell Cardiol 2005; 38: 1-2.

21. Pacary E, Heng J, Azzarelli R, Riou P, Castro D, Lebel-Potter M et al. Proneural transcription factors regulate different steps of cortical neuron migration through Rnd-mediated inhibition of RhoA signaling. Neuron 2011; 69: 1069-1084.

22. Mocholi E, Ballester-Lurbe B, Arque G, Poch E, Peris B, Guerri C et al. RhoE deficiency produces postnatal lethality, profound motor deficits and neurodevelopmental delay in mice. PLoS One 2011; 6: e19236.

23. Lin X, Liu B, Yang X, Yue X, Diao L, Wang J et al. Genetic deletion of Rnd3 results in aqueductal stenosis leading to hydrocephalus through up-regulation of Notch signaling. Proc Natl Acad Sci USA 2013; 110: 8236-8241.

24. Yang $X$, Lin $X$, Yue $X$, Chang J. Rnd3 functions as a new regulator in fetal heart arrhythmias through beta2-adrenergic receptor-PKA signaling pathway. 2013 ISHR World Congress; 2013 June 30-July 4, 2013. International Society for Heart Research: San Diego, CA, USA, 2013. p PS1-PS88.

25. Wennerberg K, Forget MA, Ellerbroek SM, Arthur WT, Burridge K, Settleman J et al. Rnd proteins function as RhoA antagonists by activating p190 RhoGAP. Curr Biol 2003; 13: 1106-1115.

26. Riento K, Villalonga $P$, Garg R, Ridley A. Function and regulation of RhoE. Biochem Soc Trans 2005; 33: 649-651.

27. Riento K, Ridley AJ. Rocks: multifunctional kinases in cell behaviour. Nat Rev Mol Cell Biol 2003; 4: 446-456.

28. Boswell SA, Ongusaha PP, Nghiem P, Lee SW. The protective role of a small GTPase RhoE against UVB-induced DNA damage in keratinocytes. J Biol Chem 2007; 282: 4850-4858.

29. Poch E, Minambres R, Mocholi E, Ivorra C, Perez-Arago A, Guerri C et al. RhoE interferes with $\mathrm{Rb}$ inactivation and regulates the proliferation and survival of the U87 human glioblastoma cell line. Exp Cell Res 2007; 313: 719-731.

30. Bektic J, Pfeil K, Berger AP, Ramoner R, Pelzer A, Schafer G et al. Small G-protein RhoE is underexpressed in prostate cancer and induces cell cycle arrest and apoptosis. Prostate 2005; 64: 332-340.

31. Chang J, Xie M, Shah VR, Schneider MD, Entman ML, Wei $L$ et al. Activation of Rho-associated coiled-coil protein kinase 1 (ROCK-1) by caspase-3 cleavage plays an essential role in cardiac myocyte apoptosis. Proc Natl Acad Sci USA 2006; 103: 14495-14500.

32. Coleman ML, Sahai EA, Yeo M, Bosch M, Dewar A, Olson MF. Membrane blebbing during apoptosis results from caspase-mediated activation of ROCK I. Nat Cell Biol 2001; 3: 339-345.

33. Sebbagh M, Renvoize C, Hamelin J, Riche N, Bertoglio J, Breard J. Caspase-3-mediated cleavage of ROCK I induces MLC phosphorylation and apoptotic membrane blebbing. Nat Cell Biol 2001; 3: 346-352.

34. Ueda H, Morishita R, Itoh H, Narumiya S, Mikoshiba K, Kato K et al. Galpha11 induces caspase-mediated proteolytic activation of Rho-associated kinase, ROCK-I, in HeLa cells. J Biol Chem 2001; 276: 42527-42533. 
35. Yang X, Li Q, Lin X, Ma Y, Yue X, Tao Z et al. Mechanism of fibrotic cardiomyopathy in mice expressing truncated Rho-associated coiled-coil protein kinase 1. FASEB J 2012; 26 2105-2116.

36. Zhang YM, Bo J, Taffet GE, Chang J, Shi J, Reddy AK et al. Targeted deletion of ROCK protects the heart against pressure overload by inhibiting reactive fibrosis. FASEB J 2006; 20: 916-925.

37. Shi J, Zhang YW, Yang Y, Zhang L, Wei L. ROCK1 plays an essential role in the transition from cardiac hypertrophy to failure in mice. J Mol Cell Cardiol 2010; 49: 819-828.

38. Rikitake Y, Oyama N, Wang CY, Noma K, Satoh M, Kim HH et al. Decreased perivascular fibrosis but not cardiac hypertrophy in ROCK1 $+/$ - haploinsufficient mice. Circulation 2005; 112: 2959-2965.

39. Amano M, Fukata Y, Kaibuchi K. Regulation and functions of Rho-associated kinase. Exp Cell Res 2000; 261: 44-51.

40. Nobes CD, Lauritzen I, Mattei MG, Paris S, Hall A, Chardin P. A new member of the Rho family, Rnd1, promotes disassembly of actin filament structures and loss of cell adhesion. J Cell Biol 1998; 141: 187-197.

41. Guasch RM, Scambler P, Jones GE, Ridley AJ. RhoE regulates actin cytoskeleton organization and cell migration. Mol Cell Biol 1998; 18: 4761-4771.

42. Fiegen D, Blumenstein L, Stege P, Vetter IR, Ahmadian MR. Crystal structure of Rnd3/ RhoE: functional implications. FEBS Lett 2002; 525: 100-104.

43. Do e Z, Fukumoto Y, Takaki A, Tawara S, Ohashi J, Nakano M et al. Evidence for Rho-kinase activation in patients with pulmonary arterial hypertension. Circ J 2009; 73: 1731-1739.

44. Belgiovine C, Frapolli R, Bonezzi K, Chiodi I, Favero F, Mello-Grand M et al. Reduced expression of the ROCK inhibitor Rnd 3 is associated with increased invasiveness and metastatic potential in mesenchymal tumor cells. PLoS One 2010; 5: e14154.

45. Ma W, Wong CC, Tung EK, Wong CM, Ng IO. RhoE is frequently down-regulated in hepatocellular carcinoma $(\mathrm{HCC}$ ) and suppresses $\mathrm{HCC}$ invasion through antagonizing the Rho/Rho-kinase/myosin phosphatase target pathway. Hepatology 2013; 57: 152-161.

46. Grise F, Sena S, Bidaud-Meynard A, Baud J, Hiriart JB, Makki K et al. Rnd3/RhoE Is down-regulated in hepatocellular carcinoma and controls cellular invasion. Hepatology 2012; 55: 1766-1775.
47. Masumoto A, Hirooka Y, Shimokawa H, Hironaga K, Setoguchi S, Takeshita A. Possible involvement of Rho-kinase in the pathogenesis of hypertension in humans. Hypertension 2001; 38: 1307-1310.

48. Hirooka Y, Shimokawa H, Takeshita A.. Rho-kinase, a potential therapeutic target for the treatment of hypertension. Drug News Perspect 2004; 17: 523-527.

49. Masumoto A, Mohri M, Shimokawa H, Urakami L, Usui M, Takeshita A. Suppression of coronary artery spasm by the Rho-kinase inhibitor fasudil in patients with vasospastic angina. Circulation 2002; 105: 1545-1547.

50. Mohri M, Shimokawa H, Hirakawa Y, Masumoto A, Takeshita A. Rho-kinase inhibition with intracoronary fasudil prevents myocardial ischemia in patients with coronary microvascular spasm. J Am Coll Cardiol 2003; 41: 15-19.

51. Vicari RM, Chaitman B, Keefe D, Smith WB, Chrysant SG, Tonkon MJ et al. Efficacy and safety of fasudil in patients with stable angina: a double-blind, placebo-controlled, phase 2 trial. J Am Coll Cardiol 2005; 46: 1803-1811.

52. Li Q, Lin X, Yang X, Chang J. NFATc4 is negatively regulated in miR-133a-mediated cardiomyocyte hypertrophic repression. Am J Physiol Heart Circ Physiol 2010; 298: $\mathrm{H} 1340-\mathrm{H} 1347$.

(c) (i) $(\Theta$ Cell Death and Disease is an open-access journal published by Nature Publishing Group. This work is licensed under a Creative Commons Attribution-NonCommercialNoDerivs 3.0 Unported License. The images or other third party material in this article are included in the article's Creative Commons license, unless indicated otherwise in the credit line; if the material is not included under the Creative Commons license, users will need to obtain permission from the license holder to reproduce the material. To view a copy of this license, visit http://creativecommons.org/licenses/ by-nc-nd/3.0/

Supplementary Information accompanies this paper on Cell Death and Disease website (http://www.nature.com/cddis) 